\title{
Questions asked through two examples of dilemmas of publication ethics in the process of COVID-19
}

\author{
Ozgur Tanriverdi ${ }^{1,2,3}$ (D) Muesser Ozcan ${ }^{3,4}$
}

Received: 18 July 2020 / Accepted: 14 August 2020 / Published online: 24 August 2020

○) Springer Science+Business Media, LLC, part of Springer Nature 2020

\begin{abstract}
The COVID-19 pandemic is a kind of global disaster caused by the new coronavirus-19, the SARS-CoV-2 virus. Since the first eruption of this pandemic, which adversely affected the world in many ways, a large number of publications have been presented to the world of science. In this article, possible publication ethical dilemmas related to scientific articles increasing in number during the COVID-19 pandemic were tried to be reminded through two examples of articles.
\end{abstract}

Keywords COVID-19 pandemic $\cdot$ SARS-Cov-2 $\cdot$ Publishing ethics $\cdot$ Scientific articles

\section{To the Editor}

Between January 2020 and June 2020, it was determined that in the "PubMed" search engine, where international scientific researches were intensely indexed, the total number of publications corresponding to the keyword "COVID-19" and "COVID-19 treatment" was 385 . All the journals about a nightmare pandemic seemed to have entered the race to quickly include scientific publications.

It is obvious that researches related to this virus-related molecular pathogenesis, prevention and treatment of disease, which affect the whole world economically, socially, psychologically, and academically, are very important for all humanity. However, the fact that many journals gave priority to the studies related to the pandemic may have caused a scientific pollution with the decision of publishing very

Ozgur Tanriverdi

dr.ozgur.tanriverdi@gmail.com

1 Department of Medical Oncology, Faculty of Medicine, Mugla Sitki Kocman University, Mugla Universitesi Egitim Ve Arastirma Hastanesi, Onkoloji Poliklinigi, 48000 Mugla, Turkey

2 Department of Molecular Biology and Genetics, Graduate School of Natural and Applied Sciences, Mugla Sitki Kocman University, Mugla, Turkey

3 Department of Elderly Health, Faculty of Health Science, Mugla Sitki Kocman University, Mugla, Turkey

4 Deparment of Medicine History and Ethics, Faculty of Medicine, Mugla Sitki Kocman University, Mugla, Turkey quickly. It was also possible that there were points that were overlooked in terms of both the reliability of the data and the filtering of violations in terms of ethics.

Indeed, the subsequent withdrawal of two separate studies published in the form of a large patient series for COVID-19 treatment reminded us of the value of these ethical dilemmas. Two scientific publications that had to be withdrawn by their authors were published in two valuable, respected and ethically sensitive journals in medicine, Lancet and the New England Journal of Medicine [1,2].

The first article was published in the May 2020 issue of the Lancet Journal titled "Hydroxychloroquine or chloroquine with or without a macrolide for treatment of COVID19: a multinational registry analysis" [1]. In this study of chloroquine and hydroxychloroquine, which caused controversy in the treatment of COVID-19, the clinical results of the patient treated with a significant number of COVID-19, such as 96032, were evaluated based on the recording system. It has been reported that chloroquine and hydroxychloroquine therapies administered alone or in combination with macrolide did not have positive results after the comparison over the four treatment arms. However, with the announcement dated June 5th, 2020, the article named by the editors of the Lancet Journal has been taken down. Despite the fact that the data accuracy, which was overlooked during the initial evaluation, was examined by an independent group as the main reason for this decision, it was stated that the authors could not meet this request and therefore there was a lack of confidence in the data [1]. 
The second article was titled "Cardiovascular disease, drug therapy, and mortality in Covid-19" by Mehra et al., published in the May 2020 issue of the New England Journal of Medicine [2]. In this study, where data from 169 hospitals and 8910 COVID-19 hospitalized patients were analyzed, it was found that the underlying cardiovascular diseases significantly increased the risk of death due to COVID-19, but there was no negative effect between ACE inhibitors and ARBs and mortality. It is quite interesting that this article was also withdrawn by the New England Journal of Medicine on 04.06.2020 since it was another work of the first author of the article that was withdrawn from the Lancet Journal. In the statement on the withdrawal of the article, there was an emphasis on the fact that not all authors have access to the raw data of the study and therefore the initial data cannot be subject to an independent review [2].

The questions to be asked ethically regarding this situation are actually important for the dilemmas of publication ethics. These questions can be listed as follows:

1. Did the authors not behave ethically in this multicenter registration study, in which the number of patients was quite good?

2. As a reputable journal hesitates about data reliability through retrospective analysis, can hesitation be experienced about the reliability of other publications related to COVID-19 in journals that cannot fully implement similar ethical principles?

3. It is noteworthy that the ethical violation is not a journal or author, but a negative result in relation to a drug in which many studies are being continued for treatment. Are there any ethical violations regarding the role of the pharmaceutical industry in withdrawing these research?

To the database of patients of other physicians without permission inclusion of patients or repeated entry of patient data without coordination among physicians can be considered as ethical violations of the authors. In this study using multi-centered data, whether physicians who are not included in the author list and whose patients are transferred to the database without permission, stated that there is an ethical violation in this regard, this issue is not clear. Another ethical dilemma assumption is whether a retrospective awareness process on the inconsistency of the data by the journal's editorial board is working. In any case, these questions may remain questions that cannot be answered within the scope of the rapid broadcasting process.

It is a possibility that journals may have accepted the publications with the fast publishing system in the first months of the pandemic's paralysis of the whole life and the scientific world. Considering that there are countless journals related to medicine and health sciences, the effect of this rapid publishing process will be noticeable years later. This question, unfortunately, is a question that cannot be answered.

Despite the rigidity of the drug studies monitoring and ethical conditions, due to sectoral intervention, the comment pollution of these two publications may have caused the withdrawal of the articles. However, this is a question that will take place only in the science archive, like other questions that cannot be answered.

As a result, it is very important to design scientific articles that share effective or ineffective treatment methods in the treatment of a lethal disease in accordance with the rules of publication [3].

\section{References}

1. Mehra MR, Ruschitzka F, Patel AN. Hydroxychloroquine or chloroquine with or without a macrolide for treatment of COVID-19: a multinational registry analysis. Lancet. 2020;395:1820.

2. Mehra MR, Desai SS, Kuy SR, Henry TD, Patel AN. Cardiovascular disease, drug therapy, and mortality in Covid 19. New Engl J Med. 2020;382:e102.

3. Gollogly L, Momen H. Ethical dilemmas in scientific publication: pitfalls and solutions for editors. Rev Saude Publ. 2006;40:20-9.

Publisher's Note Springer Nature remains neutral with regard to jurisdictional claims in published maps and institutional affiliations. 